\title{
A new method for hardness determination from depth sensing indentation tests
}

\author{
J. Gubicza, A. Juhász, and J. Lendvai \\ Department of General Physics, Eötvös University, Budapest, H-1088 Múzeum krt. 6-8, \\ Budapest, Hungary
}

(Received 18 December 1995; accepted 30 July 1996)

\begin{abstract}
A new semiempirical formula is developed for the hardness determination of the materials from depth sensing indentation tests. The indentation works measured both during loading and unloading periods are used in the evaluation. The values of the Meyer hardness calculated in this way agree well with those obtained by conventional optical observation, where this latter is possible. While the new hardness formula characterizes well the behavior of the conventional hardness number even for the ideally elastic material, the mean contact pressure generally used in hardness determination differs significantly from the conventional hardness number when the ideally elastic limiting case is being approached.
\end{abstract}

Hardness testing with sharp indenters is generally considered as a simple method for characterizing the mechanical strength of materials. Recently the depth sensing indentation (DSI) test became a widely used method of hardness determination. ${ }^{1-8}$ In the DSI tests the applied load is registered as a function of indentation depth both during loading and unloading. A schematic load versus penetration depth curve is shown in Fig. 1. The most frequently used DSI method was developed by Oliver and Pharr ${ }^{3}$ by which the hardness number can be determined without optical observation. In this method the mean contact pressure at the maximum depth is used to characterize the plastic properties of materials. If, however, a considerable part of the deformation during the indentation is elastic, this pressure deviates significantly from the conventional hardness number which is determined by optical observation of the indentation trace. For example, in the limiting case of the ideally elastic material, the conventional hardness number tends to infinity while the mean contact pressure gives finite value since an elastic contact surface is developing between the indenter tip and the ideally elastic material. ${ }^{2}$

The paper is a continuation of a recently published work $^{9}$ in which a new semiempirical formula has been developed for the determination of the Meyer hardness ${ }^{10}$ of materials. With the formula proposed, even the limiting case of the ideally elastic materials can be correctly described. The main results of our recent paper are briefly summarized below to give a basis for the subsequent comparison with another evaluation method. ${ }^{3}$

Hardness measurements were carried out on different materials by the DSI method using a computercontrolled hydraulic mechanical testing machine with a Vickers indenter. During the loading period the Vickers pyramid penetrates the sample at constant velocity, and the same velocity is applied in the unloading period when the pyramid moves backwards. In the course of the test, the load is registered as a function of the penetration depth. The measurements were carried out in the macrohardness region $\left(P_{m} \approx 100 N\right)$ on the following materials: metals (99.99\% pure $\mathrm{Al}$ and $\mathrm{Cu}$ ), soda lime silica glass, sodium chloride, polypropylene, and $\mathrm{Si}_{3} \mathrm{~N}_{4}$ ceramics of two compositions sintered to different densities. Compositions and densities of ceramic samples shown in Table I.

The conventional definition of the Meyer hardness for any sharp indenter geometry is the following ${ }^{10}$ :

$$
H=\frac{P}{A},
$$

where $P$ is the applied load and $A$ is the residual projected area of the hardness impression after unloading. The hardness measurement was originally developed for testing metals in which the deformation is mostly plastic $\left(\sigma_{y} / E\right.$ is small where $\sigma_{y}$ is the flow stress and $E$ is Young's modulus); therefore, there is practically no elastic recovery under unloading, and the projected area at the maximum depth equals the residual projected area after unloading. Consequently, the mean contact pressure at the maximum penetration depth (defined as the indentation load divided by the projected contact area) equals the conventional hardness number $(H)$ determined after unloading. This is also the case when the elastic portion of the deformation during the indentation is negligible and the projected area before unloading agrees well with that measured after unloading, because - although the elastic recovery may be significant for the indentation depth-the characteristic surface dimensions exhibit only minor recovery. ${ }^{1,10}$ On the other hand, if the deformation is mostly elastic 


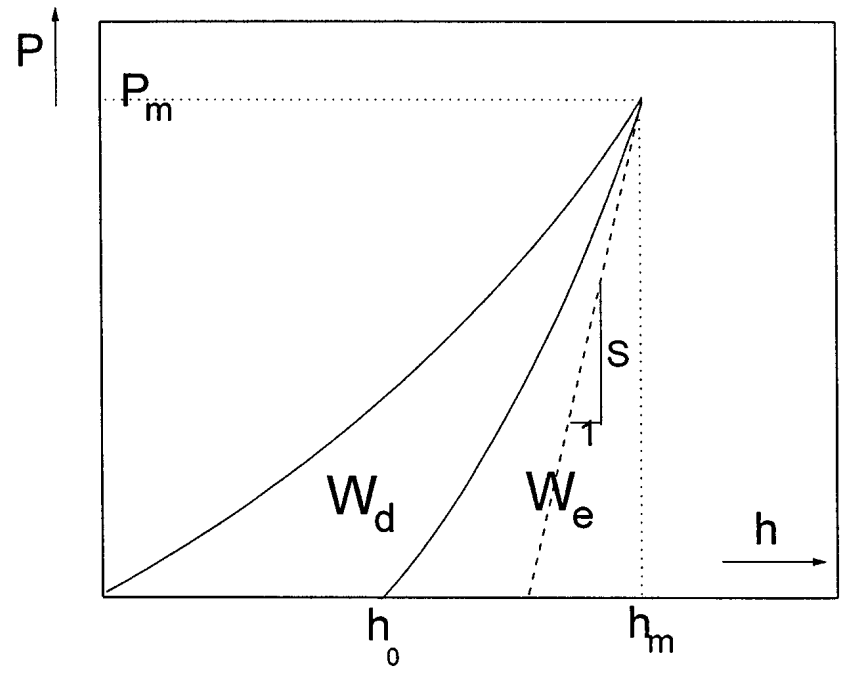

FIG. 1. Schematic picture of an indentation cycle.

TABLE I. Compositions and densities of $\mathrm{Si}_{3} \mathrm{~N}_{4}$ ceramic samples.

\begin{tabular}{ccccc}
\hline \hline \multicolumn{2}{c}{$\begin{array}{c}90 \text { wt. \%, } \mathrm{Si}_{3} \mathrm{~N}_{4}, 4 \text { wt. \% } \\
\mathrm{Al}_{2} \mathrm{O}_{3}, 6 \text { wt. \% } \mathrm{Y}_{2} \mathrm{O}_{3}\end{array}$} & & \multicolumn{2}{c}{$\begin{array}{c}90.9 \text { wt. \%, } \mathrm{Si}_{3} \mathrm{~N}_{4}, 3 \text { wt. \% } \\
\mathrm{Al}_{2} \mathrm{O}_{3}, 6.1 \text { wt. \% } \mathrm{Y}_{2} \mathrm{O}_{3}\end{array}$} \\
\cline { 1 - 2 } \cline { 5 - 6 } Sample & Density $\left(\mathrm{g} / \mathrm{cm}^{3}\right)$ & & Sample & Density $\left(\mathrm{g} / \mathrm{cm}^{3}\right)$ \\
\hline 1 & 2.03 & & 6 & 2.697 \\
2 & 2.11 & & 7 & 2.823 \\
3 & 2.34 & & 8 & 2.935 \\
4 & 2.54 & & 9 & 2.954 \\
5 & 2.70 & & 10 & 3.032 \\
& & 11 & 3.115 \\
& & 12 & 3.161 \\
\hline \hline
\end{tabular}

( $\sigma_{y} / E$ is large) then a significant portion of the contact area at maximum depth is due to elastic deformation. Consequently, the residual projected area is smaller than the projected contact area at maximum penetration, and the conventional Meyer hardness is larger than the mean contact pressure.

The load-penetration depth function can be described with quadratic polinoms (Fig. 1):

$$
\begin{aligned}
& P=c_{2} h+c_{3} h^{2}, \\
& P=c_{2}^{*}\left(h-h_{0}\right)+c_{3}^{*}\left(h-h_{0}\right)^{2},
\end{aligned}
$$

both in the loading and in the unloading periods, respectively, where $P$ is the load, $h$ is the penetration depth, and $h_{0}$ is the residual indentation depth after removing the punch; $c_{2}, c_{3}, c_{2}^{*}$, and $c_{3}^{*}$ are fitting parameters. The total indentation work, $W_{t}$, is the integral of the load versus the indentation depth, i.e., the area under the load-penetration depth curve during the unloading period. Upon unloading a part of this work, $W_{e}$, can be regained; it equals to the area under the load-indentation depth curve for this latter period. The difference of these two quantities, $W_{d}$, gives the net work expended during the entire loading-unloading period (Fig. 1):

$$
W_{d}=W_{t}-W_{e} .
$$

The work performed during loading $\left(W_{t}\right)$ and that regained during unloading $\left(W_{e}\right)$ can be calculated by the integration of Eqs. (2) and (3), respectively.

It was found that in spite of the linear terms appearing in Eqs. (2) and (3) for a broad variety of materials and in a wide load range, the following relationship is with good accuracy valid (see Fig. 2):

$$
\frac{W_{e}}{W_{t}}=\sqrt{\frac{c_{3}}{c_{3}^{*}}} .
$$

The parameter $c_{3}$ characterizes the resistance of the material against the elastic-plastic deformation. ${ }^{11}$ In the case of ideally plastic materials, the load-depth function is purely quadratic: $P=c_{3} h^{2}, 1,12$ and in this case there is no elastic relaxation, the $d=7 \cdot h_{m}$ equation between the diagonal $d$ and the maximum indentation depth $h_{m}$ which is the consequence of the geometry of the Vickers pyramid is exactly satisfied [Fig. 3(a)]. Consequently the Meyer hardness of ideally plastic materials can be given in the following form:

$$
H=2 \frac{P}{d^{2}}=\frac{P}{24.5 h_{m}^{2}}=\alpha_{1} \cdot c_{3}
$$

with $\alpha_{1}=0.0408$ for the Vickers geometry.

If the material is not ideally plastic then with increasing elastic contribution, the elastic deflection under the indenter is increasing [Fig. 3(b)]. Consequently $7 h_{m}$ becomes increasingly larger than $d$, and as a result of this $\alpha_{1} \cdot c_{3}$ will be less than $H$. This can be taken into account introducing a relationship between $H$ and $\alpha_{1} \cdot c_{3}$ of the form:

$$
H=\alpha_{1} \cdot c_{3} \cdot \frac{W_{t}}{W_{d}} .
$$

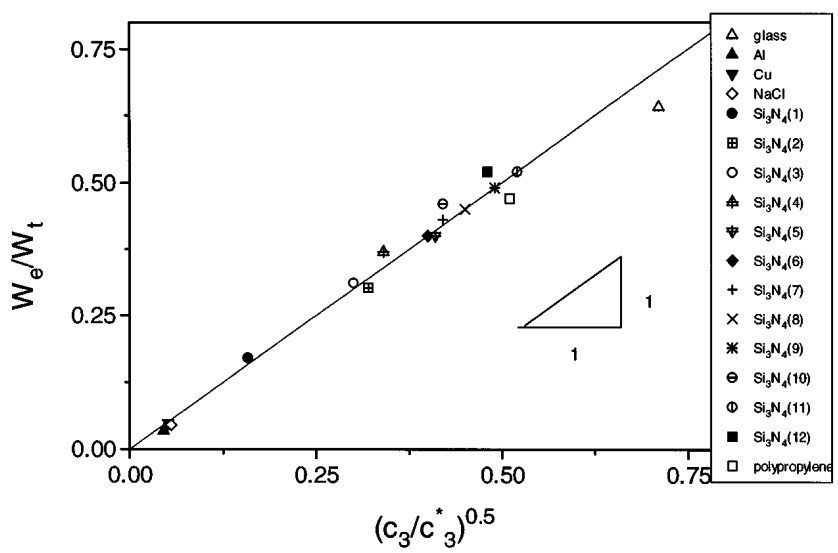

FIG. 2. The ratio of the elastic and total work versus the parameters of the indentation curves. 


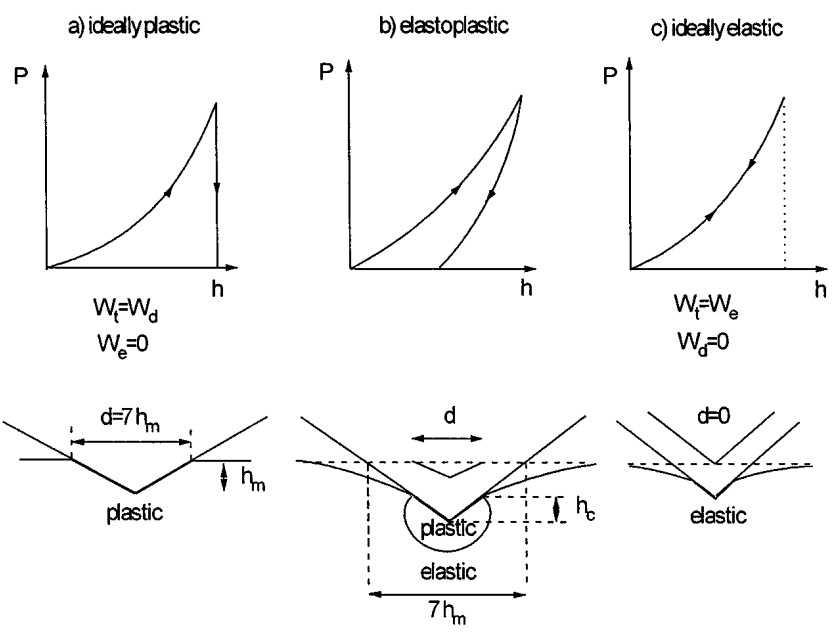

FIG. 3. Schematic picture showing the behavior of various materials $(a-c)$ during Vickers indentation.

It is obvious that this new definition of $H$ gives back Eq. (6) for ideally plastic materials and it increases with increasing elasticity, and in the limiting case of an ideally elastic material it becomes infinite, because in this case there is no residual deformation after unloading [Fig. 3(c)]. Figure 4 shows that the $H$ values determined from DSI measurements according to Eq. (7) agree well within the experimental errors with the conventionally determined hardness.

The most frequently used hardness determination method from DSI measurements introduced by Oliver and $\mathrm{Pharr}^{3}$ is based on the mean contact pressure at the maximum indentation depth with the following expression:

$$
H_{m}=\frac{P_{m}}{A}=\frac{P_{m}}{24.5 h_{c}^{2}}=\alpha_{1} \frac{P_{m}}{h_{c}^{2}},
$$

where $A$ is the projected contact area and $h_{c}$ is the contact depth at the maximum load [Fig. 3(b)]. Using

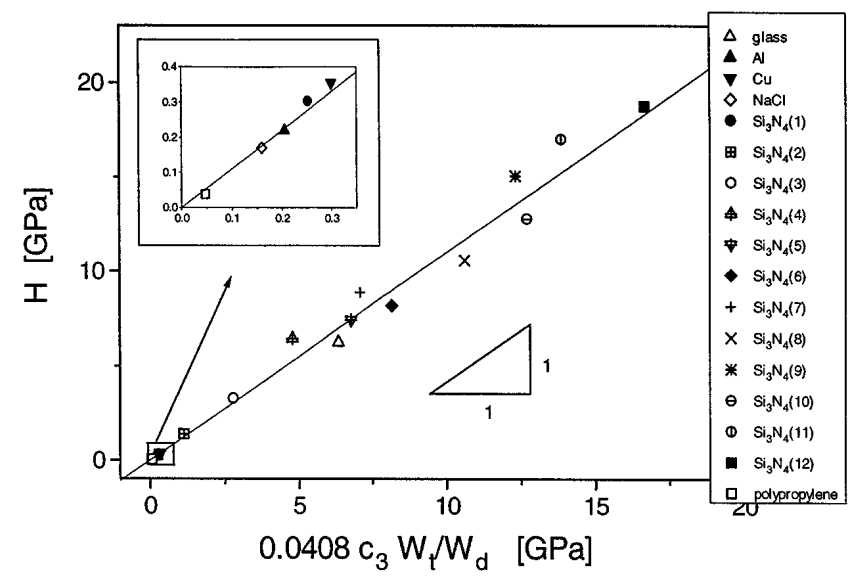

FIG. 4. The conventionally determined hardness number versus the quantity calculated on the basis of Eq. (7).
Sneddon's elastic theory ${ }^{13}$ and empirical results of Oliver and Pharr, ${ }^{3}$ the contact depth can be given as:

$$
h_{c}=h_{m}-\epsilon \frac{P_{m}}{S},
$$

where $S$ is the slope of the initial part of the unloading curve (Fig. 1) and $\epsilon=0.75$ for the case of Vickers indenters.

To compare the new hardness formula (7) with the mean contact pressure as expressed in Eqs. (8) and (9), $P_{m}$ and $h_{c}$ are expressed with the $c_{3}$ and $c_{3}^{*}$ parameters and the indentation works. If only the quadratic terms existed in Eqs. (2) and (3), $P_{m}$ and $h_{c}$ could be expressed easily with the parameters $c_{3}$ and $c_{3}^{*}$. Because of the existence of the linear terms, some approximations are used in the considerations.

According to Eq. (3) $S$ can be given as

$$
S=\left.\frac{d P}{d h}\right|_{h_{m}}=c_{2}^{*}+2 c_{3}^{*}\left(h_{m}-h_{0}\right) .
$$

The second term in Eq. (10) may be expressed as a fraction of $S$ :

$$
\bar{k} S=2 c_{3}^{*}\left(h_{m}-h_{0}\right)
$$

and similarly the quadratic term of the load-depth function of the unloading curve as a fraction of the maximum load:

$$
k^{*} P_{m}=c_{3}^{*}\left(h_{m}-h_{0}\right)^{2} .
$$

With Eqs. (11) and (12) $P_{m} / S$ can be given as

$$
\frac{P_{m}}{S}=\frac{\bar{k}}{k^{*}} \frac{k^{*}}{\bar{k}} \frac{h_{m}-h_{0}}{2} .
$$

From Eqs. (3) and (10)-(13), $h_{c}$ can be given in the following form:

$$
h_{c}=h_{m}-\frac{2}{1+k^{*}} \frac{\epsilon}{2}\left(h_{m}-h_{0}\right) .
$$

Expressing the quadratic term in Eq. (2) as a fraction of the maximum load:

$$
k P_{m}=c_{3} h_{m}^{2},
$$

the $H_{m}$ mean contact pressure in Eq. (8) can be written as follows:

$$
H_{m}=\alpha_{1} c_{3} \frac{1}{\left(\sqrt{k}-\frac{2 \sqrt{k^{*}}}{1+k^{*}} \frac{\epsilon}{2} \sqrt{\frac{c_{3}}{c_{3}^{*}}}\right)} .
$$

According to our measurements for the different materials investigated with different loads, $k$ and $k^{*}$ vary between 0.64 and 1 . If $k^{*} \geqslant 0.64$ then $\left(2 \sqrt{k^{*}}\right) /(1+$ $\left.k^{*}\right) \geqslant 0.98$; therefore, this quantity can be taken as 1 . 
Using Eq. (5) the following relationship is obtained for $H_{m}$ :

$$
H_{m}=\alpha_{1} c_{3} \frac{1}{\left(\sqrt{k}-\frac{\epsilon}{2} \frac{W_{e}}{W_{t}}\right)^{2}} .
$$

This can be compared with the new hardness formula Eq. (7), which using Eq. (4) can be written as:

$$
H=\alpha_{1} c_{3} \frac{1}{1-\frac{W_{e}}{W_{t}}} .
$$

The difference between $H$ and $H_{m}$ can be seen in Fig. 5 in which the two hardness numbers divided by $\alpha_{1} c_{3}$ are shown as a function of $W_{e} / W_{t}$. For $H_{m} / \alpha_{1} c_{3}$ three curves are shown as $\sqrt{k}$ equal to 0.8 or 0.9 or $1(k=0.64$ or 0.81 or 1$) . W_{e} / W_{t}$ equals zero for the ideally plastic limiting case; it increases with increasing elastic deformation in the indentation, and it is 1 for the ideally elastic material. By the new hardness formula [Eqs. (7) or (18)], the behavior of the conventional hardness number (and consequently the plastic properties of materials) is better characterized than by the mean contact pressure, because while the former tends to infinity the latter gives a finite value in the ideally elastic limiting case. As it can be seen in Fig. 5 in the region $0 \leqslant$ $W_{e} / W_{t} \leqslant 0.7$, there is no significant difference between the two hardness numbers. The $W_{e} / W_{t}$ values for the materials investigated here are in this region; consequently the hardness numbers obtained by both methods agree well with the values of the Meyer hardness obtained by optical observation after unloading (Fig. 5).

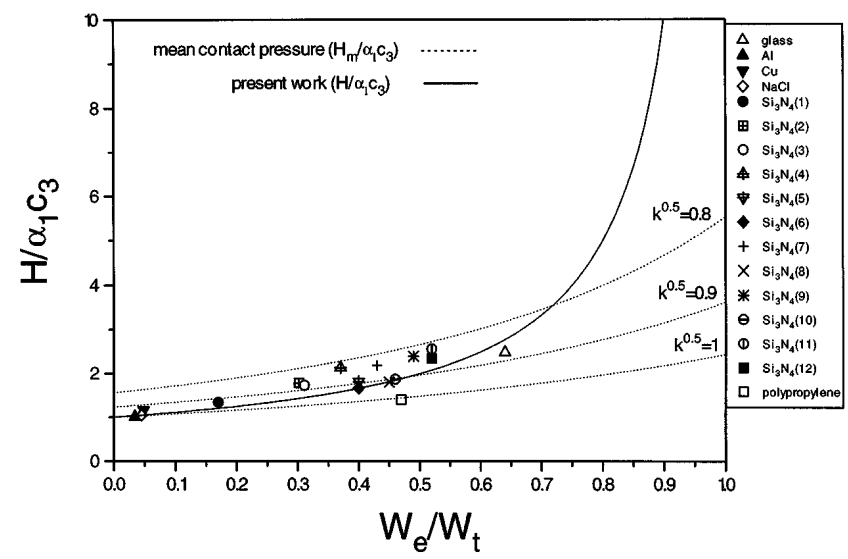

FIG. 5. The hardness numbers divided by $\alpha_{1} c_{3}$ as a function of $W_{e} / W_{t}$.
A new formula is proposed for the characterization of the hardness of materials from DSI tests. The values of the hardness calculated by this equation agree well with those measured by the conventional method for a broad variety of materials. Comparing the new hardness formula with that based on the mean contact pressure, the former correctly describes the behavior of the conventional hardness both in the ideally plastic and ideally elastic limiting cases, while the latter deviates from the conventional hardness number in the ideally elastic limit. At the same time in the range where optical measurements can also be applied $\left(0 \leqslant W_{e} / W_{t} \leqslant 0.7\right)$, the difference between the two DSI evaluation methods is within the experimental error. A technical advantage of the new evaluation method is that the parameters used in the hardness formula can be determined at a high accuracy from the registered indentation curves. Further measurements are planned on materials and for loading conditions falling in the region where the new hardness number is rapidly increasing $\left(0.8 \leqslant W_{e} / W_{t} \leqslant 1\right)$. This regime, however, is not easy to obtain experimentally.

\section{ACKNOWLEDGMENTS}

The authors are grateful to P. Arató for providing the $\mathrm{Si}_{3} \mathrm{~N}_{4}$ ceramic samples. This work was supported by the Hungarian National Scientific Fund in Contract Nos. T-017637 and T-017639.

\section{REFERENCES}

1. M. Sakai, Acta Metall. Mater. 41, 1751 (1993).

2. G. M. Pharr, W.C. Oliver, and F. R. Brotzen, J. Mater. Res. 7, 613 (1992).

3. W. C. Oliver and G. M. Pharr, J. Mater. Res. 7, 1564 (1992).

4. G. M. Pharr and W. C. Oliver, MRS Bull. 17, 28 (1992).

5. A. Juhász, G. Vörös, P. Tasnádi, I. Kovács, I. Somogyi, and J. Szöllösi, Colloque C7, supplément au J. de Phys. 3, 1485 (1993).

6. F. R. Brotzen, Int. Mat. Rev. 39, 24 (1994).

7. A. Juhász, M. Dimitrova-Lukács, G. Vörös, J. Gubicza, P. Tasnádi, P. Lukács, and A. Kele, Fortchrittsberichte der Deutschen Keramischen Gesellschaft 9, 87 (1994).

8. J. Gubicza, Key Eng. Mater. 103, 217 (1995).

9. J. Gubicza, A. Juhász, P. Arató, P. Tasnádi, and G. Vörös, J. Mater. Sci. 31, 3109 (1996).

10. D. Tabor, Hardness of Metals (Clarendon Press, Oxford, 1951).

11. F. Fröhlich, P. Grau, and W. Grellmann, Phys. Status Solidi (a) 42, 79 (1977).

12. B. R. Lawn and V. R. Howes, J. Mater. Sci. 16, 2745 (1981).

13. I. N. Sneddon, Int. J. Engng. Sci. 3, 47 (1965). 